Cite this: Dalton Trans., 2014, 43 , 5134

Received 14th December 2013, Accepted 30th January 2014

DOI: 10.1039/c3dt53512f

www.rsc.org/dalton

\section{Variable photon energy photoelectron spectroscopy of tris-cyclopentadienyl lanthanides}

\author{
Marcello Coreno, ${ }^{a}$ Monica de Simone, ${ }^{\mathrm{b}}$ Jennifer C. Green, ${ }^{\text {c }}$ Nikolas Kaltsoyannis, ${ }^{d}$ \\ Rosemary Coates, ${ }^{d}$ Charlene Hunston, ${ }^{d}$ Naima Narband ${ }^{d}$ and Andrea Sellad
}

The gas phase photoelectron (PE) spectra of $\mathrm{LnCp}_{3}\left(\mathrm{Cp}=\eta-\mathrm{C}_{5} \mathrm{H}_{5} ; \mathrm{Ln}=\mathrm{Pr}, \mathrm{Nd}, \mathrm{Sm}\right)$, measured with a wide range of photon energy, are reported. Resonances observed in the photon energy regions of $4 \mathrm{~d}$ to $4 \mathrm{f}$ excitation enable identification of ion states resulting from $4 \mathrm{f}$ ionization. For all three compounds molecular ion states characteristic of both $4 \mathrm{f}^{n}$ and $4 \mathrm{f}^{n-1}$ configurations are observed ( $\mathrm{Pr}, n=2 ; \mathrm{Nd}, n=3 ; \mathrm{Sm}$, $n=6$ ). The molecular ion ground states have a hole in the uppermost ligand orbital of a' symmetry and are reached by either ligand or $f$ electron ionization. The results are discussed in the context of the previously reported spectra of the $\mathrm{Ce}, \mathrm{Yb}$ and $\mathrm{Lu}$ analogues. For $\mathrm{YbCp}_{3} \mathrm{f}$ orbital/ligand interaction is proposed in the molecular ground state and for $\mathrm{CeCp}_{3}{ }^{+}$in the molecular ion ground state. For $\operatorname{PrCp} 3$ and $\mathrm{NdCp}_{3}$ final state effects are proposed as the origin of the dual configuration structure in their PE spectra. When the contributing orbitals are close in energy the 4f/a' interaction can give rise to significant covalent bonding even in the absence of effective overlap.
For d block transition metal complexes, gas phase photoelectron (PE) spectroscopy has had considerable success in revealing their electronic structure. For closed shell molecules in the valence region, there is normally a close correspondence between the photoelectron bands and the orbital structure predicted by density functional theory (DFT) ${ }^{1,2}$ Lanthanide complexes are not so straightforward. The f-electrons are energetically part of the valence region, and for some elements are active in redox chemistry, but spatially are part of the core, the f-orbitals lacking significant overlap with ligand orbitals. Core ionization may result in significant perturbation of a molecule's electronic structure, which manifests itself in satellite structure accompanying the primary core PE bands, often referred to as shake-up or shake-down bands. These bands are assigned to many electron processes in which a valence excitation or transition accompanies the core ionization.

Ionization of the $4 \mathrm{f}$ electrons of lanthanides gives very characteristic final state structure that depends on the electronic ground state adopted by the $4 \mathrm{f}^{n}$ configuration. The relative intensities of the ion states arising from ionization of the uf electrons have been predicted using a fractional parentage description of the ground state molecule, ${ }^{3}$ and these

\footnotetext{
${ }^{a}$ CNR-IMIP, Montelibretti, Rome I-00016, Italy

${ }^{b}$ CNR-IOM, Laboratorio TASC, 34149 Trieste, Italy

${ }^{c}$ Department of Chemistry, Oxford University, South Parks Road, Oxford OX1 $3 Q R$,

UK. E-mail: jennifer.green@chem.ox.ac.uk

${ }^{d}$ Department of Chemistry, University College London, 20 Gordon Street,

London WC1H OAJ, UK
}

predictions are on the whole consistent with data obtained from solid state photoemission studies. ${ }^{4}$ Although consideration of spin orbit coupling leads to modification of the relative intensity predictions, ${ }^{5}$ the pattern of the bands associated with a particular $4 \mathrm{f}^{n-1}$ configuration are characteristic and may be assigned by consideration of the electronic spectrum of the $\mathrm{Ln}^{3+}$ ion of the preceding element, i.e. the lanthanide with an atomic number of one less and a $4 \mathrm{f}^{n-1}$ configuration. $^{6}$

The cross sections of $4 \mathrm{f}$ electrons differ substantially from those of the higher lying ligand-based electrons, which are predominantly of $\mathrm{p}$ character. ${ }^{7}$ The $4 \mathrm{f}$ cross sections have a delayed maximum in the photon energy region between 75 and $150 \mathrm{eV}$ depending on the particular lanthanide. By contrast, the cross sections of $\mathrm{s}$ and $\mathrm{p}$ bands have maxima close to threshold, hence employing incident photon energies away from $\mathrm{p}$ based maxima yet near to $4 \mathrm{f}$ maxima will enable unambiguous characterization of the final state structure. The $4 \mathrm{f}$ bands also exhibit cross section maxima in the region of the $4 \mathrm{~d}$ to $4 \mathrm{f}$ resonant excitation. ${ }^{2,8,9}$ Such excitation opens another channel for photoionization, since the excitation can be followed by super Coster-Kronig (SCK) decay in which the core hole is refilled and an $\mathrm{f}$ electron is ejected. Thus, in a PE experiment, with careful selection of photon energies, as is enabled by synchrotron radiation, ion states accessible by $\mathrm{f}$ ionization may be distinguished from those accessible by ligand ionization.

PE studies of lanthanides and their compounds in the solid state normally show f ionization bands superimposed on the valence bands. In some cases, particularly intermetallic 
compounds and those with counterions of low electronegativity, $\mathrm{f}$ ionization bands corresponding to two different $\mathrm{f}$ configurations are found. Thus, if the compound has a formal oxidation state implying a $4 \mathrm{f}^{n}$ configuration, both $4 \mathrm{f}^{n-1}$ and $4 \mathrm{f}^{n}$ band patterns are observed. Such observations have been interpreted in a number of ways. In some cases the initial state is implicated and a mixture of oxidation states is proposed for the compound. ${ }^{10}$ In other cases the $4 \mathrm{f}^{n}$ states are presumed to result from ionization of an $f$ electron being accompanied by valence band to $4 \mathrm{f}$ electron transfer i.e. a final state effect. ${ }^{11,12}$

Gas phase PE studies on lanthanide complexes are less common than those of solid compounds. This is in part due to their low volatility but also because discharge sources, common in house, are of insufficient energy to be able to identify unambiguously $4 \mathrm{f}$ ionization bands. The availability of an angle resolved photoelectron spectrometer (ARPES) on the gas phase photoemission beam line of the Elettra synchrotron $^{13}$ has enabled a programme of study of the tris-cyclopentadienyl lanthanides, $\operatorname{LnCp}_{3}\left(\mathrm{Cp}=\eta-\mathrm{C}_{5} \mathrm{H}_{5}\right)$. We have reported so far on the PE spectra of $\mathrm{CeCp}_{3},{ }^{14,15} \mathrm{YbCp}_{3}{ }^{6}$ and $\mathrm{LuCp}_{3} .{ }^{6}$ The PE spectrum of $\mathrm{LuCp}_{3}$ shows two ion states, ${ }^{2} \mathrm{~F}_{7 / 2}$ and ${ }^{2} \mathrm{~F}_{5 / 2}$ associated with a $4 \mathrm{f}^{13}$ configuration for the molecular ion as might be anticipated on ionizing the closed $4 \mathrm{f}^{14}$ shell of $\mathrm{Lu}^{3+}$. The PE spectrum of $\mathrm{YbCp}_{3}$ shows structure corresponding to both $4 \mathrm{f}^{12}$ and $4 \mathrm{f}^{13}$ ion configurations. Consequently it was proposed that $\mathrm{YbCp}_{3}$ has a mixed configuration ground state that can be represented by the superposition of an ionic configuration $\mathrm{Yb}(\mathrm{III}): 4 \mathrm{f}^{13}\left(\mathrm{Cp}_{3}\right)$ and a charge-transfer configuration $\mathrm{Yb}(\mathrm{II}): 4 \mathrm{f}^{14}\left(\mathrm{Cp}_{3}\right)^{-1}$. Subsequent confirmation was obtained by the study of the magnetism, epr spectrum and analysis of the electronic spectrum of $\mathrm{YbCp}_{3}$, which were all in agreement in weighting the charge transfer configuration in the range $12-17 \% .{ }^{16}$ In addition, the hole in the ligand set in the charge transfer configuration was found to be in an orbital of a' symmetry ( $C_{3 \mathrm{~h}}$ molecular symmetry) which interacts with one of the real parts of the $4 \mathrm{f}_{ \pm 3}$ orbitals. In the case of $\mathrm{CeCp}_{3}$ the $\mathrm{PE}$ spectrum also indicated two ion state configurations, but in this case it is the molecular ion that has a mixed configuration ground state, $\mathrm{Ce}(\mathrm{Iv}): 4 \mathrm{f}^{0}\left(\mathrm{Cp}_{3}\right)$ mixed with $\mathrm{Ce}(\mathrm{III}): 4 \mathrm{f}^{1}\left(\mathrm{Cp}_{3}\right)^{-1}$. The molecular ion ground state is accessible by direct photoionization from the $\mathrm{Ce}(\mathrm{III}): 4 \mathrm{f}^{1}\left(\mathrm{Cp}_{3}\right)$ molecular configuration by virtue of the former term. ${ }^{14}$ Calculations using a CASSCF/CASPT2 approach confirmed the presence of configuration interaction in the ground state of the $\mathrm{CeCp}_{3}{ }^{+}$cation. ${ }^{15}$

Here we present the variable photon energy PE spectra of $\mathrm{PrCp}_{3}, \mathrm{NdCp}_{3}$ and $\mathrm{SmCp}_{3}$ and compare the results with those reported previously for $\mathrm{Ce}, \mathrm{Yb}$ and $\mathrm{Lu}$.

\section{Experimental}

The tris-cyclopentadienyl lanthanides of praseodymium, neodymium and samarium were prepared by the method of Birmingham and Wilkinson and sublimed at $200{ }^{\circ} \mathrm{C} / 10^{-2} \mathrm{mmHg}$ to remove coordinated $\mathrm{THF}^{17}$ The resulting product was sealed in a glass ampoule and subsequently transferred to a copper container under nitrogen, which was inserted into an oven attached to the angle resolved photoelectron spectrometer.

Measurements were carried out at the GAs PHase photoemission (GAPH) beam line of the ELETTRA storage ring in Trieste, Italy. ${ }^{13}$ An undulator source provides high-intensity synchrotron radiation in the photon energy range 20-900 eV. The highly polarized light is dispersed by a Variable Angle Spherical Grating Monochromator that is equipped with five interchangeable gratings, to cover the energy range 13 to 900 $\mathrm{eV}$, and fixed entrance and exit slits, with a photon energy resolution $\Delta E / E \leq 10^{-4}$.

The samples were held in an oven close to the ionization region and evaporated through a small hole by holding the temperature of the oven at around $161^{\circ} \mathrm{C}$.

The PE measurements on $\mathrm{NdCp}_{3}$ were carried out using the ARPES chamber. The ARPES chamber was equipped with a $50 \mathrm{~mm}$ mean radius electron energy analyser (VSW Ltd), mounted at the magic angle. Pass energies of $10 \mathrm{eV}$ were used for valence PE spectra at low photon energies and $15 \mathrm{eV}$ at higher photon energies. The PE spectra of $\mathrm{PrCp}_{3}$ and $\mathrm{SmCp}_{3}$ were analyzed by a VG220i hemispherical electron energy analyzer with a mean radius of $150 \mathrm{~mm}$ equipped with six channel electron multipliers. This electron analyser was mounted in the plane defined by the (linearly polarized) electric vector of the light and the photon propagation direction at an angle of $54.7^{\circ}$ with respect to the electric vector of the light. In this geometry the axis of the analyzer is set at the pseudo magic angle, and so measurements should be insensitive to the PE asymmetry $\beta$ parameter. Electron energy resolution is linear along all the kinetic energy scale and equal to $2 \%$ of pass band for both analyzers. PE spectra were acquired at the magic angle or pseudo magic angle depending on the analyzer used and normalised using the signal from a calibrated photodiode (IRD, Inc.). ${ }^{18}$ Pass energies of $5 \mathrm{eV}$ were used for valence PE spectra. PE spectra of the valence region from $6-20 \mathrm{eV}$ of binding energy were measured at the pseudo magic angle for a series of photon energies ranging from 24-180 eV. The binding energy scale was calibrated according to the Ar photoelectron lines.

\section{Results}

A selection of the PE spectra of $\mathrm{LnCp}_{3}(\mathrm{Ln}=\mathrm{Pr}, \mathrm{Nd}, \mathrm{Sm})$ measured with both low and higher photon energies are shown in Fig. 1-3.

Key features are tabulated (Table 1). The ionization bands fall into two distinct types. Those labelled A-G, identifiable for all three compounds, show a decay in intensity relative to the other bands with increasing photon energy. (Although only two photon energy spectra are presented in the figures, many more were recorded which show a gradual evolution.) Those bands labelled with roman numerals increase in relative intensity with photon energy and show resonances in the photon energy region of the respective lanthanide $4 \mathrm{~d}$ to $4 \mathrm{f}$ excitation 


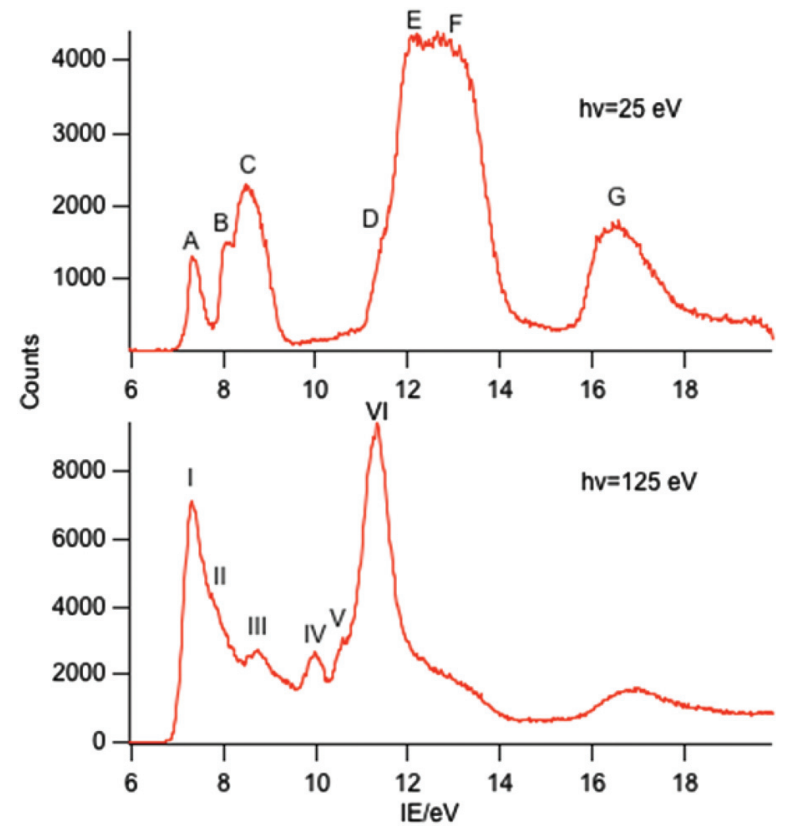

Fig. 1 PE spectra of $\mathrm{PrCp}_{3}$ with photon energies of 25 and $125 \mathrm{eV}$.

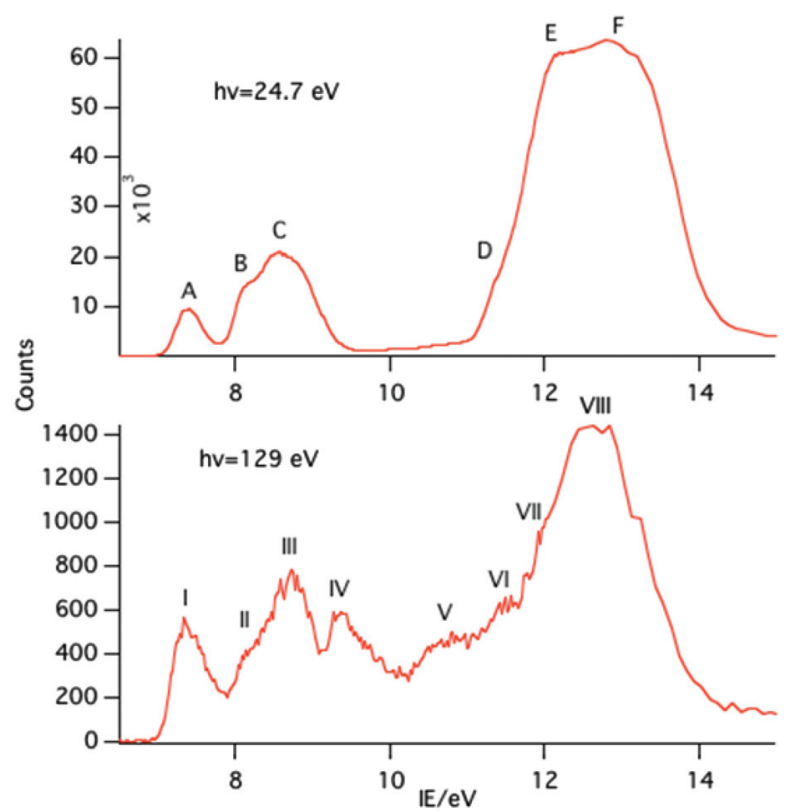

Fig. 2 PE spectra of $\mathrm{NdCp}_{3}$ with photon energies of 24.7 and $129 \mathrm{eV}$.

energies. The emergence of the f-bands over the resonance region is shown for $\mathrm{PrCp}_{3}$ in Fig. 4. Although the $\mathrm{f}$ bands dominate in the resonance region they are also visible in the preresonance region for example with a photon energy of $100 \mathrm{eV}$.

In the spectra of all three compounds bands $\mathrm{A}$ and I are coincident indicating that the ground ion states are accessible by two different ionization processes.

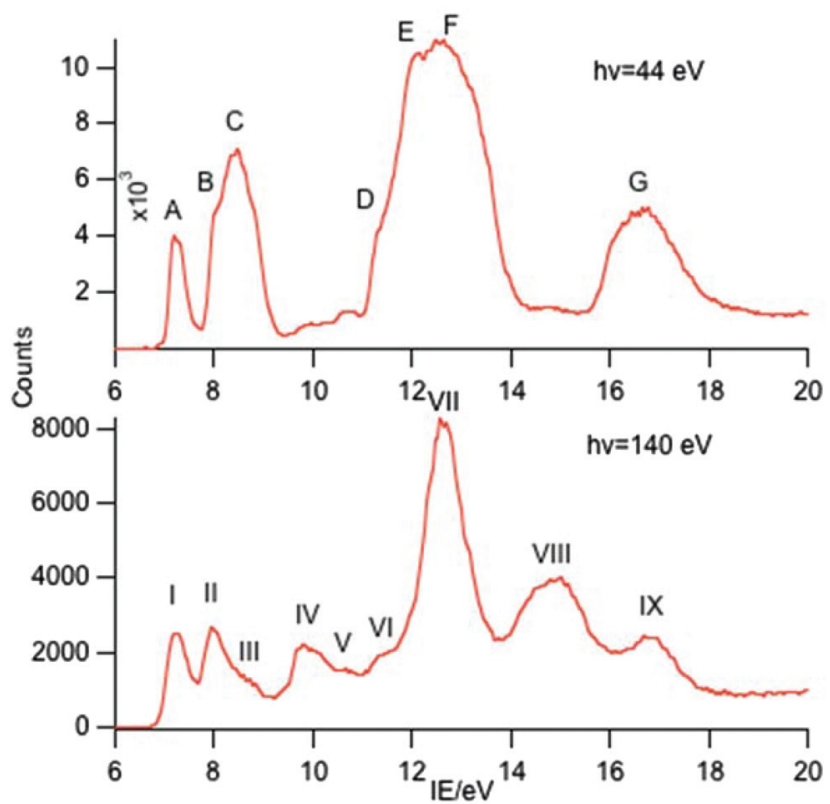

Fig. 3 PE spectra of $\mathrm{SmCp}_{3}$ with photon energies of 44 and $140 \mathrm{eV}$.

\section{Discussion}

\section{Band assignment}

The origin of the bands $\mathrm{A}-\mathrm{G}$ and their assignment has been discussed previously. ${ }^{6,14-16}$ The associated ion states have holes in the ligand shells and are accessed from the parent molecules by ionization of ligand based orbitals. PE ionization cross sections of carbon and hydrogen based electrons have a maximum near threshold and decay with increasing photon energy, ${ }^{2,7}$ thus the ready identification of their ionization bands. Individual assignments are based on comparison with the PE spectra of other cyclopentadienyl compounds and theoretical calculations.

Most crucially bands A, B and C arise from ionization of the highest occupied $\pi$ orbitals of the cyclopentadienyl rings. In the $C_{3 \mathrm{~h}}$ symmetry of the gas-phase $\mathrm{LnCp}_{3}$ molecules these transform as $\mathrm{a}^{\prime}+\mathrm{a}^{\prime \prime}+\mathrm{e}^{\prime}+\mathrm{e}^{\prime \prime}$. The Ln $5 \mathrm{~d}$ transform as $\mathrm{a}^{\prime}, \mathrm{e}^{\prime}$, and $\mathrm{e}^{\prime \prime}$, and the $6 \mathrm{p}$ as a" and $\mathrm{e}^{\prime}$. However, the net overlap of the cylindrically symmetrical $5 d_{z^{2}}\left(a^{\prime}\right)$ with the Cp- $\pi\left(a^{\prime}\right)$ MO, which has six nodes in the horizontal plane (Fig. 5), is negligible; in the effective $D_{3 \mathrm{~h}}$ symmetry they would belong to different irreducible representations. The $\mathrm{Cp}-\pi$ a' combination does find a nodal match in the $\cos 3 \phi$ component of the $\operatorname{Ln} 4 \mathrm{f}_{ \pm 3}$ orbitals but lack of substantive radial overlap with the core-like $4 \mathrm{f}$ orbitals leads to it being less stabilized by interaction with the lanthanide than the other combinations, which can overlap with Ln $5 d$ and $6 p$ orbitals.

Band A is assigned to ionization from this $\mathrm{Cp}-\pi$ a' orbital and lies at a lower IE, well separated from bands B and C. Band B is assigned to ionization from the Cp- $\pi$ a" orbital which can mix with Ln $5 p$ and band $C$ to the $\mathrm{e}^{\prime}$ and $\mathrm{e}^{\prime \prime}$ orbitals which form more effective bonding combinations with Ln $5 \mathrm{~d}$ 
Table 1 Ionization energies (eV) of key features in the PE spectra of $\mathrm{LnCp}_{3}\left(\mathrm{Ln}=\mathrm{Ce},{ }^{14} \mathrm{Pr}, \mathrm{Nd}, \mathrm{Sm}, \mathrm{Yb}^{6}{ }^{6} \mathrm{Lu}^{6}\right)$

\begin{tabular}{|c|c|c|c|c|c|c|c|}
\hline Band $\backslash$ Ln & $\mathrm{Ce}$ & $\operatorname{Pr}$ & $\mathrm{Nd}$ & $\mathrm{Sm}$ & $\mathrm{Yb}$ & $\mathrm{Lu}$ & Associated MO \\
\hline A & 7.45 & 7.34 & 7.39 & 7.21 & 7.16 & 7.28 & $a^{\prime}$ \\
\hline B & 8.13 & 8.04 & 8.17 & 8.08 & & & $a^{\prime \prime}$ \\
\hline D & 11.53 & 11.46 & 11.57 & 11.50 & & & \\
\hline $\mathrm{E}$ & 12.25 & 12.19 & 12.22 & 12.07 & 12.4 & 12.4 & \\
\hline F & 12.80 & 12.94 & 12.79 & 13.00 & & & \\
\hline G & 16.53 & 16.49 & 16.53 & 16.60 & 16.7 & 17.2 & \\
\hline II & 9.97 & 7.76 & 8.14 & 7.96 & 8.58 & 15.74 & \\
\hline III & & 8.77 & 8.74 & 8.61 & 12.93 & & \\
\hline IV & & 9.97 & 9.39 & 9.86 & 13.73 & & \\
\hline V & & 10.61 & 10.79 & 10.66 & 14.91 & & \\
\hline VI & & 11.34 & 11.49 & 11.46 & 15.98 & & \\
\hline VII & & & 11.99 & 12.56 & 16.71 & & \\
\hline
\end{tabular}

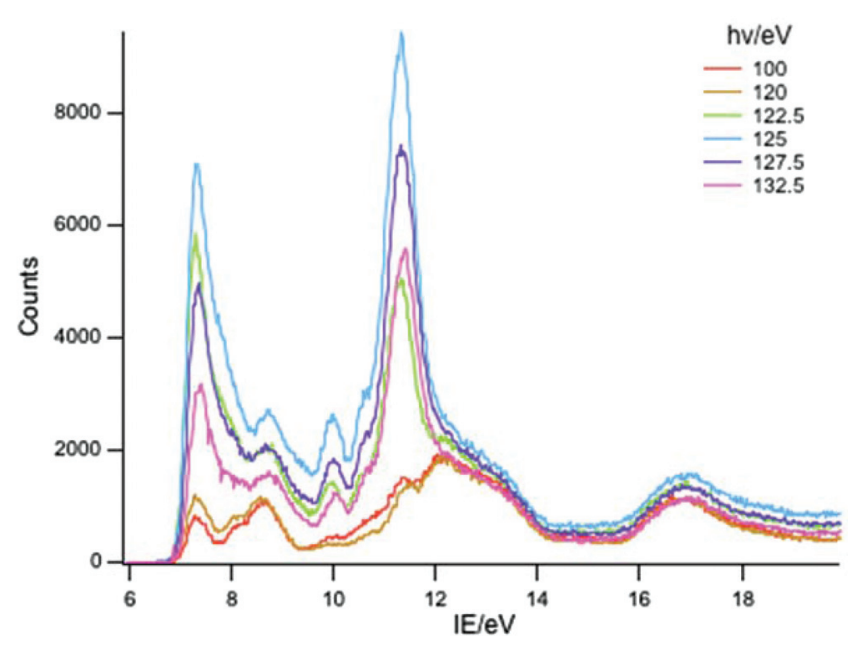

Fig. 4 PE spectra of $\operatorname{PrCp}_{3}$ with photon energies 100-132.5 eV.

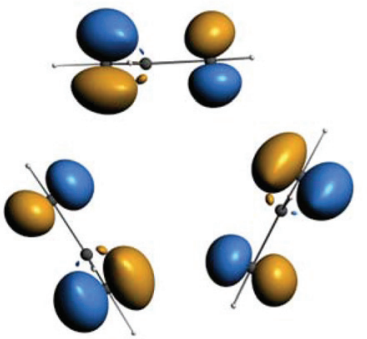

(a)

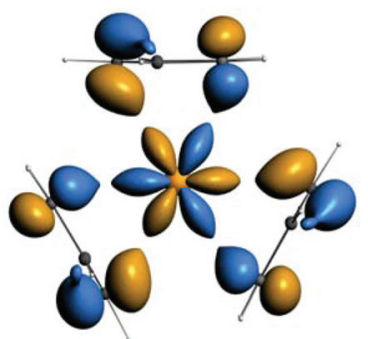

(b)
Fig. 5 (a) The $\mathrm{Cp}-\pi$ a' symmetry adapted linear combination (b) possible

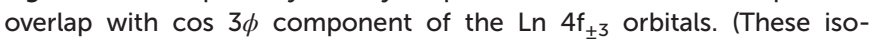
surfaces at value 4.8 were generated for $\mathrm{CeCp}_{3}{ }^{+}$using the ADF program suite BP/TZP. $)^{19}$

orbitals. Bands D-F are associated with orbitals based on C $2 \mathrm{p}$ and $\mathrm{H} 1 \mathrm{~s}$ orbitals whereas band $\mathrm{G}$ is principally $\mathrm{C} 2 \mathrm{~s}$ in character.
As discussed in the introduction, the cross sections of $4 \mathrm{f}$ ionizations are very low near threshold with the consequence that the associated ionization bands are not readily detectable with low photon energies. ${ }^{2,7}$ They rise in intensity with photon energy passing through a maximum. In addition $4 \mathrm{f}$ ionizations commonly show resonances when the photon energy promotes $4 \mathrm{~d}$ to $4 \mathrm{f}$ excitation. Intensity borrowing from this transition may lead to considerable enhancement of a $4 \mathrm{f}$ band intensity. ${ }^{2}$ The ionizations labelled with roman numerals show such cross section features and are associated with ionizations from the $4 \mathrm{f}$ orbitals. The number and intensity of the $4 \mathrm{f}$ bands is characteristic of the number of $f$ electrons in the molecule.

$\mathbf{P r C p}_{3}$. Fig. 6 shows a difference spectrum of $\mathrm{PrCp}_{3}$ consisting of the pre-resonance spectrum at $117.5 \mathrm{eV}$ subtracted from that at the height of the resonance at $125 \mathrm{eV}$. This process removes the ligand bands from the spectrum leaving only the $\mathrm{f}$ band spectrum. $\mathrm{Pr}^{3+}$ has a $4 \mathrm{f}^{2}$ configuration and a ${ }^{3} \mathrm{H}_{4}$ ground state. Fractional parentage methods predict that on direct photoionization the spin orbit states ${ }^{2} \mathrm{~F}_{5 / 2}$ and ${ }^{2} \mathrm{~F}_{7 / 2}$ are accessed with relative intensity 1.714 to 0.286 . The spin orbit

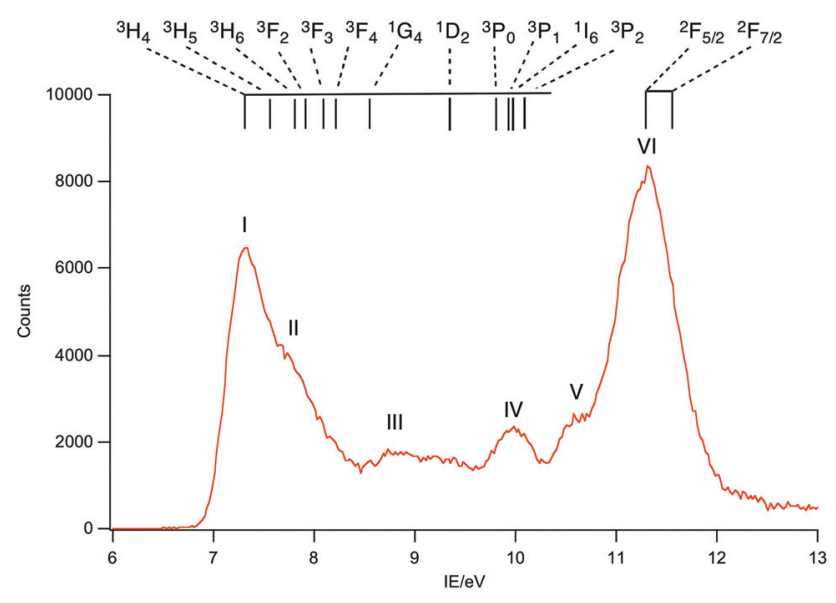

Fig. 6 Difference of PE spectrum at $125 \mathrm{eV}$ minus $\mathrm{PE}$ spectrum at $110 \mathrm{eV}$ for $\mathrm{PrCp}_{3}$. The highest energy $4 \mathrm{f}$ band, $\mathrm{VI}$, is assigned to the ${ }^{2} \mathrm{~F}$ states. 
splitting of $\operatorname{Pr}^{4+}$ is $865 \mathrm{~cm}^{-1}(0.1 \mathrm{eV})^{20}$ so resolution of these states is not expected under the experimental conditions. Thus the presence of six $4 \mathrm{f}$ bands in the PE spectrum indicates that simple direct photoionization from a $\mathrm{Pr}^{3+}$ ion is not the only process to be considered.

Band I, being coincident with band A, corresponds to an ion state with a hole in the Cp- $\pi$ a' orbital and $\operatorname{Pr}\left(\right.$ III) in its ${ }^{3} \mathrm{H}_{4}$ ground state. We denote this ion state $\left|4 \mathrm{f}^{2}\left({ }^{3} \mathrm{H}_{4}\right) \mathrm{a}^{\prime-1}\right\rangle$. Bands II-V are assigned to excited states of the $4 \mathrm{f}^{2}$ configuration and are assigned by comparison with electronic spectra of $\operatorname{Pr}(\mathrm{III})$ compounds and associated calculations. ${ }^{21,22}$ Such calculations include consideration of the crystal field, and that splitting of the spin-orbit states can cover an energy range of up to $0.2 \mathrm{eV}$. In Fig. 6 we indicate the relative energies of the lowest crystal field term of a particular spin orbit state of $\operatorname{Pr}^{3+}$. It is evident that the spin-orbit states span the energy range of bands I-V.

$\mathbf{N d C p}_{3}$. Fig. 7 shows a difference spectrum of $\mathrm{NdCp}_{3}$ consisting of the pre-resonance spectrum at $110 \mathrm{eV}$ subtracted from that at the height of the resonance at $129 \mathrm{eV}$. $\mathrm{Nd}$ (III) with an $\mathrm{f}^{3}$ configuration and a ${ }^{4} \mathrm{I}_{9 / 2}$ ground state is predicted to give ${ }^{3} \mathrm{H}_{4},{ }^{3} \mathrm{H}_{5}$ and ${ }^{3} \mathrm{~F}_{2}$ states upon direct photoionization with relative intensities $1.890: 0.424: 0.563$. In $\mathrm{PrCp}_{3}^{*}\left(\mathrm{Cp}^{*}=\eta-\mathrm{C}_{5} \mathrm{Me}_{5}\right)$ these states span an energy range of $0.7 \mathrm{eV}^{21}$ For $\mathrm{Nd}^{4+}$ an expansion of the range is anticipated but all three states are likely to occur within the $1.5 \mathrm{eV}$ range covered by band VIII, which is consequently assigned to these ${ }^{3} \mathrm{H}_{4},{ }^{3} \mathrm{H}_{5}$ and ${ }^{3} \mathrm{~F}_{2}$ states.

Like $\mathrm{PrCp}_{3}, \mathrm{NdCp}_{3}$ has many more $4 \mathrm{f} \mathrm{PE}$ bands than can be accounted for by direct photoionization. The lowest energy $4 \mathrm{f}$ band, I, is again coincident with the lowest energy ligand band, A, and can be assigned to the molecular ion ground state $\left|4 \mathrm{f}^{3}\left({ }^{4} \mathrm{I}_{9 / 2}\right) \mathrm{a}^{-1}\right\rangle$. Nd(III) has a plethora of spin-orbit states. In Fig. 7 we indicate the energy range of identified states for $\mathrm{Nd}^{3+} \cdot{ }^{23,24}$ Bands II-VII all lie within this range.

$\mathrm{SmCp}_{3}$. Fig. 8 shows a difference spectrum for $\mathrm{SmCp}_{3}$ consisting of a pre-resonance spectrum acquired at $130 \mathrm{eV}$ subtracted from one at the height of the resonance at $140 \mathrm{eV}$.

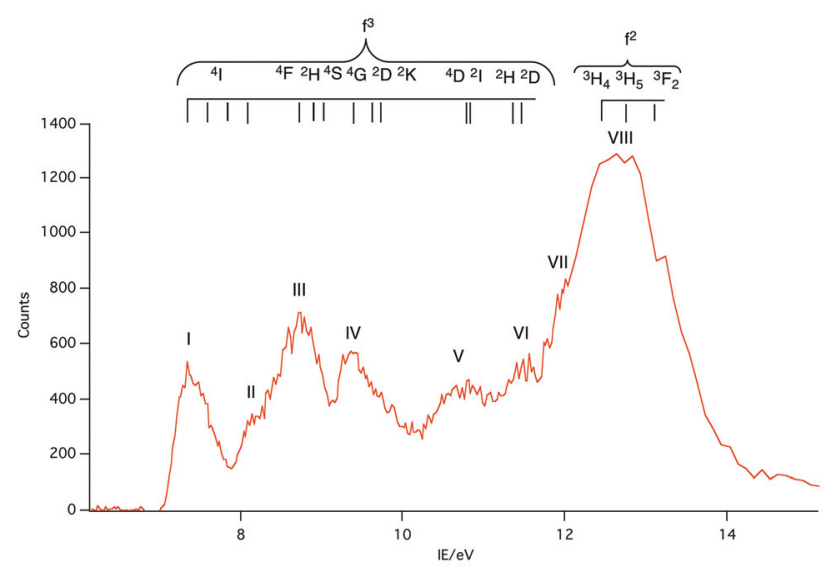

Fig. 7 Difference of PE spectrum at $129 \mathrm{eV}$ minus PE spectrum at 110 $\mathrm{eV}$ for $\mathrm{NdCp}_{3}$ showing the energy range of identified $4 \mathrm{f}^{3}$ spin-orbit states for $\mathrm{Nd}^{3+}$ and shows the likely energy range of $4 \mathrm{f}^{2}$ states for $\mathrm{NdCp}_{3}{ }^{+}$accessible by direct photoionization.

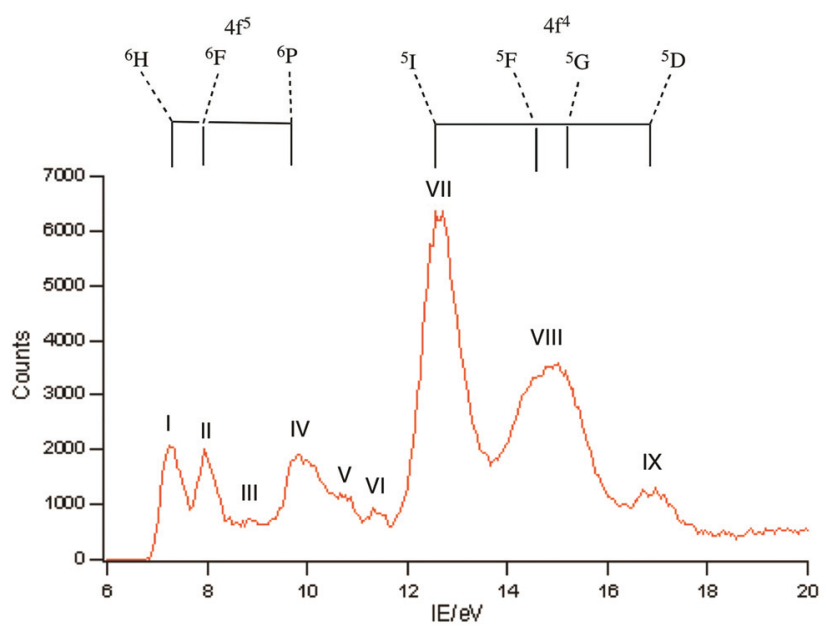

Fig. 8 Difference of PE spectrum at $140 \mathrm{eV}$ minus PE spectrum at 130 $\mathrm{eV}$ for $\mathrm{SmCp}_{3}$. The lower energy $4 \mathrm{f}$ bands are assigned to states from a $4 \mathrm{f}^{5}$ configuration and those attributed to accessible sextet states from a $4 \mathrm{f}^{4}$ configuration are labelled with their ${ }^{(2 S+1)} \mathrm{L}$ symbols. Bands III, V and $\mathrm{VI}$ are likely to arise from the many quartet states of $\mathrm{Sm}^{3+}$.

Sm(III) has a $4 \mathrm{f}^{5}$ configuration and a ${ }^{6} \mathrm{H}_{5 / 2}$ ground state. Direct ionization is predicted to give four LS states, ${ }^{5} \mathrm{I},{ }^{5} \mathrm{G},{ }^{5} \mathrm{~F}$ and ${ }^{5} \mathrm{D}$ with relative intensities $2.758: 1.266: 0.500: 0.476 .^{3}$ Bands VI, VII and VIII have relative intensities $2.76: 2.1: 0.13$ suggesting that band VII incorporates both the ${ }^{5} \mathrm{~F}$ and ${ }^{5} \mathrm{G}$ states as has been assigned previously. ${ }^{25}$ Bands I, II and IV are clearly visible in the spectra of $\mathrm{Sm}$ (II) compounds and are assigned to the ${ }^{6} \mathrm{H},{ }^{6} \mathrm{~F}$ and ${ }^{6} \mathrm{P}$ states of the $4 \mathrm{f}^{6}$ configuration..$^{10,25-27}$ Other states from this configuration are also visible as bands III, V and VI which to the best of our knowledge haven't been reported previously in photoemission studies. Assignment of these states is difficult. There is a plethora of excited states for the $\mathrm{Sm}^{3+}$ ion. Various quartet states lie between the ${ }^{6} \mathrm{~F}$ and ${ }^{6} \mathrm{P}$ levels and above the ${ }^{6} \mathrm{P}$ levels ${ }^{28}$ and we hesitate to make a definite assignment.

\section{Electronic structure and state access}

It is helpful at this juncture to propose two orbital schemes for the ground states of tris-cyclopentadienyl lanthanide systems (Fig. 9). Both include as the basis set the highest occupied cyclopentadienyl $\pi$ orbitals, the lanthanide $4 \mathrm{f}, 5 \mathrm{~d}$ and $6 \mathrm{p}$ orbitals. Mixing with the $5 \mathrm{~d}$ and $6 \mathrm{p}$ orbitals stabilizes the $\mathrm{a}^{\prime \prime}, \mathrm{e}^{\prime}$ and $\mathrm{e}^{\prime \prime}$ linear combination of the ring orbitals. Although, formally, in the $C_{3 \mathrm{~h}}$ symmetry of the molecule the ring a' orbital may interact with the $\mathrm{d}_{z^{2}}$ orbital, their nodal properties are incompatible (Fig. 5). In the first case (Fig. 9a) the 4 f orbitals are sufficiently separated in energy from the ring a' orbitals, such that the interaction is not applicable. This is the case in $\mathrm{CeCp}_{3}$.

In the second case (Fig. 9b) the $4 \mathrm{f}$ orbitals are sufficiently close in energy to the ring a' orbital for interaction and charge transfer to take place resulting in a stabilized ring orbital and a destabilized f orbital, specifically one of the real components of the $f_{ \pm 3}$ pair. It is noteworthy in the latter case that even with minimal overlap as a consequence of the radially contracted $4 \mathrm{f}$ 


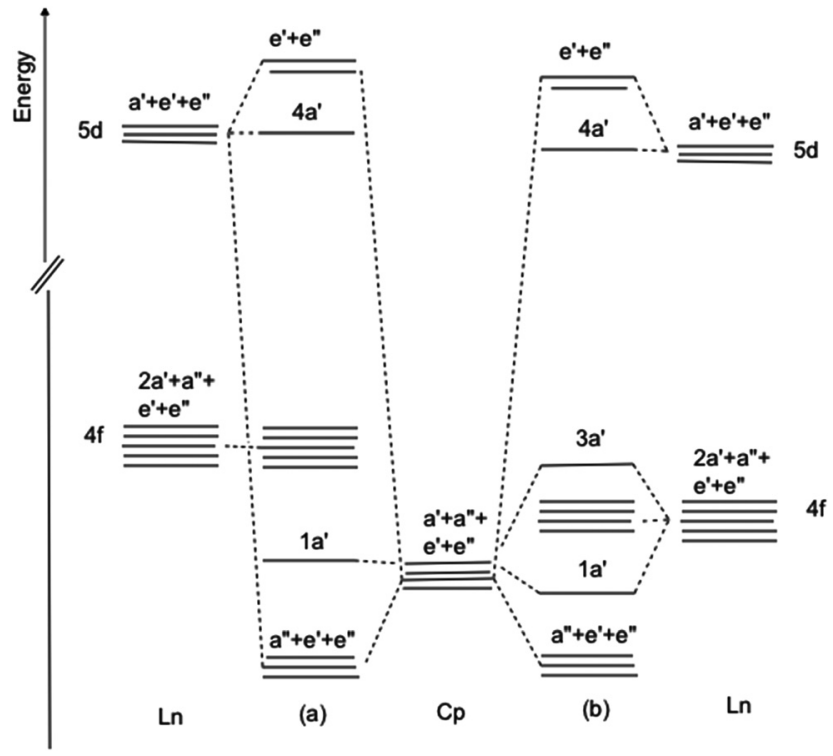

Fig. 9 Orbital interaction diagram for $\mathrm{LnCp}_{3}$ (a) $4 \mathrm{f}$ orbitals non-interacting (b) $4 \mathrm{f}$ orbitals close in energy to ring $\pi$ a' orbital.

orbitals significant interaction may occur when the interacting orbitals are close in energy. ${ }^{29}$ Case $9 \mathrm{~b}$ pertains to $\mathrm{CeCp}_{3}{ }^{+}$ cation and $\mathrm{YbCp}_{3}$. For $\mathrm{CeCp}_{3}{ }^{+} 1 \mathrm{a}^{\prime}$ is filled and is the HOMO while for $\mathrm{YbCp}_{3} 3 \mathrm{a}^{\prime}$ is the SOMO occupied by one unpaired electron.

The resulting ground state wave function is most conveniently described by configuration interaction between two terms (L represents the remaining electrons) where one configuration has $n \mathrm{f}$ electrons as expected for a $\operatorname{Ln}^{x+}$ ion and the other has a hole in the ring a' orbital and one more $f$ electron.

$$
\Psi_{\mathrm{GS}}=c_{0} \psi_{0}+c_{1} \psi_{1}=c_{0}\left|\mathrm{f}^{n} \mathrm{a}^{\prime 2} \mathrm{~L}\right\rangle+c_{1}\left|\mathrm{f}^{n+1} \mathrm{a}^{\prime 1} \mathrm{~L}\right\rangle
$$

The two terms must have the same symmetry so the crucial $4 \mathrm{f}$ orbital is of a' symmetry as shown in Fig. $5 \mathrm{~b}$.

For the $\mathrm{LnCp}_{3}{ }^{+}$cations the PE spectra inform as to the relative energies of the ground and excited states. In the cases of $\mathrm{Ln}=\mathrm{Ce}, \mathrm{Pr}, \mathrm{Nd}, \mathrm{Sm}, \mathrm{Yb}$ and $\mathrm{Lu}$ the states associated with an $\mathrm{f}^{n-1} \mathrm{a}^{\prime 2} \mathrm{~L}$ configuration, where one $\mathrm{f}$ electron has been ionized, are unambiguously identified. Removal of a $4 \mathrm{f}$ electron results in significant stabilization of the other $f$ electrons and states from this configuration lie at higher energies than the molecular ion ground state. For $\mathrm{Ln}=\mathrm{Pr}, \mathrm{Nd}, \mathrm{Sm}, \mathrm{Yb}$ and $\mathrm{Lu}$ the cationic ground states correspond simply to the removal of one electron from the Cp $\pi$ a' orbital and belong to the configuration $\mathrm{f}^{n} \mathrm{a}^{1} \mathrm{~L}$. For $\mathrm{Ln}=\mathrm{Ce}$ there is a lower energy cationic ground state with a mixed configuration as described by eqn (1). The ionization energies of the lowest states associated with a configuration are plotted in Fig. 10.

The ionization energy to the $\mathrm{f}^{n} \mathrm{a}^{\prime 1} \mathrm{~L}$ configuration remains fairly constant across the series; removal of an electron from the $\mathrm{Cp} \pi \mathrm{a}^{\prime}$ orbital is insensitive to the Ln nuclear charge. In contrast the ionization energy to the $\mathrm{f}^{n-1} \mathrm{a}^{\prime 2} \mathrm{~L}$ configuration rises across the series because the $4 \mathrm{f}$ electrons are not

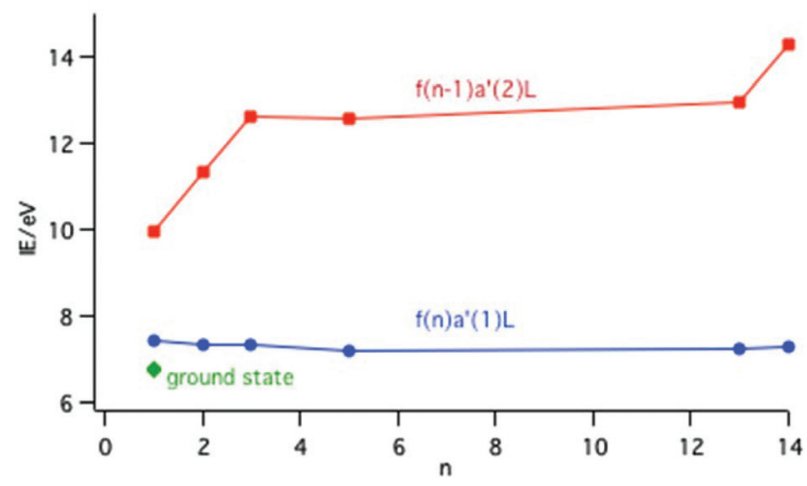

Fig. 10 Plot of the IE to the lowest energy state of a configuration across the lanthanide series; red points are associated with an $f^{n-1} a^{\prime 2} L$ configuration and blue points with an $\mathrm{f}^{n} \mathrm{a}^{\prime 1} L$ configuration. The green point represents the ground state of $\mathrm{CeCp}_{3}{ }^{+}$.

effectively shielded from the increase in Ln nuclear charge. Calculations on the $\mathrm{CeCp}_{3}{ }^{+}$cation have shown that in the $\mathrm{f}^{0} \mathrm{a}^{\prime 2} \mathrm{~L}$ state there is a considerable increase in the population of the metal $5 \mathrm{~d}$ orbitals compared with the ground state as the ligand metal covalency increases with $\mathrm{f}$ ionization. ${ }^{15}$ This is described as screening in solid state studies.

The ground state of $\mathrm{CeCp}_{3}{ }^{+}$is $0.68 \mathrm{eV}\left(65 \mathrm{~kJ} \mathrm{~mol}^{-1}\right)$ more stable than the excited state with an $\mathrm{f}^{1} \mathrm{a}^{11} \mathrm{~L}$ configuration. This separation provides a convenient measure of the binding energy afforded by the configuration interaction (CI) described by eqn (1). Delocalizing the $\mathrm{f}$ electron into the hole in the ligand $a^{\prime}$ orbital rather than maintaining pure $f$ character provides a significant covalent interaction. Modeling of the electronic spectrum of $\mathrm{YbCp}_{3}$ gave a CI matrix element of a similar order of magnitude, namely $-3900 \mathrm{~cm}^{-1}\left(47 \mathrm{~kJ} \mathrm{~mol}^{-1}\right) .{ }^{16}$ Thus in these situations of close energy proximity significant covalent binding can occur with minimal overlap.

The matrix element governing the band intensity for direct $\mathrm{f}$ ionization is given by

$$
\left\langle\operatorname{Lf}^{n}|O| \operatorname{Lf}^{n-1} \varepsilon\right\rangle
$$

where $O$ is the dipole moment operator and $\varepsilon$ is the free electron wave. Thus the $\mathrm{f}^{n-1} \mathrm{a}^{\prime 2} \mathrm{~L}$ configuration may be accessed by direct removal of an $f$ electron as can the ground state of the $\mathrm{CeCp}_{3}{ }^{+}$cation as a result of its mixed configuration. For $\mathrm{Ln}=$ $\mathrm{Pr}, \mathrm{Nd}, \mathrm{Sm}$ and $\mathrm{Yb}$ the $\mathrm{f}^{n} \mathrm{a}^{{ }^{1}} \mathrm{~L}$ configuration can also be accessed by $4 \mathrm{f}$ ionization. The question arises as to whether this is an initial or final state effect. For $\mathrm{YbCp}_{3}$ it has been shown conclusively that the neutral molecule has a mixed configuration ground state with a value for $c_{1}{ }^{2}$ of $0.12 \pm 0.03$. An alternative mechanism by which the states associated with an $\mathrm{f}^{n} \mathrm{a}^{\prime 1} \mathrm{~L}$ configuration can be accessed is ionization coupled with electronic relaxation in the ion; an electron moves from the $\mathrm{Cp}$ $\pi$ a' orbital into the stabilized $4 \mathrm{f}$ shell. Such processes are commonly found for core ionizations. Chemical intuition suggests that such a shake down is more likely for $\mathrm{Ln}=\mathrm{Pr}$ and $\mathrm{Nd}$ whereas $\mathrm{SmCp}_{3}$ may have a mixed configuration ground state ( $\mathrm{Sm}$ can form compounds in the II oxidation state). 
Consideration of charge transfer energies results in the prediction that $\mathrm{SmCp}_{3}$ may have between 3 and $4 \% \mathrm{Sm}$ (II) character. ${ }^{16}$ However, the intensities of the bands belonging to the $4 \mathrm{f}^{6}$ configuration constitute $28 \%$ of the total $4 \mathrm{f}$ band intensity, thus it is unlikely that all access to this configuration is a consequence of a mixed configuration ground state. Other techniques for probing the f orbital covalency that explicitly avoid major perturbation to the ground state are epr and variable temperature magnetism. However these are normally made on solid samples. In the solid state $\mathrm{SmCp}_{3}$ has an infinite chain structure, ${ }^{30}$ unlike $\mathrm{YbCp}_{3}$, which has a molecular crystal structure with the molecule possessing $C_{3 \mathrm{~h}}$ symmetry. ${ }^{31}$ With lower symmetry the a' ligand orbital may interact with the metal $d$ orbitals and be lowered in energy. Thus its energy proximity to the metal f orbitals will change. Given the covalency is energy driven this is likely to change between the gas phase and the solid state. In addition the electronic configuration of $\mathrm{SmCp}_{3}$ is not a Kramer's doublet, unlike $\mathrm{YbCp}_{3}$. Thus magnetic and epr measurements that were able to establish the mixed configuration ground state for $\mathrm{YbCp}_{3}$ are not accessible for $\mathrm{SmCp}_{3} \cdot{ }^{6,16} \mathrm{Sm}$ LIII/II-XANES involves major perturbations to the Sm core hence, like PES, is likely to perturb the valence electronic structure. From the PE results we cannot rule out $\mathrm{f}$ orbital covalency but the magnitude of the intensity of the $\mathrm{f}^{n}$ bands indicates that such covalency is not the cause of their presence.

\section{Conclusions}

The gas phase PE spectra of $\operatorname{LnCp}_{3}\left(\mathrm{Cp}=\eta-\mathrm{C}_{5} \mathrm{H}_{5} ; \mathrm{Ln}=\mathrm{Pr}, \mathrm{Nd}\right.$, $\mathrm{Sm})$ have been measured using synchrotron radiation. Variation of band intensity with photon energy, including in the region of $4 \mathrm{~d}$ to $4 \mathrm{f}$ excitation, has enabled unambiguous assignment of the ion states. In all three cases, ion states associated with both $4 \mathrm{f}^{n}$ and $4 \mathrm{f}^{n-1}$ configurations are identified, where $n$ is the number of $f$ electrons in the molecular ground state. The data are discussed in the context of the previous results for $\mathrm{Ln}=\mathrm{Ce}, \mathrm{Yb}$ and Lu . The cationic ground state of $\mathrm{LnCp}_{3}$ for all members of the series except Ce has a $\mathrm{f}^{n}$ configuration with a hole in the ligand shell, specifically in an orbital of a' symmetry. Ce is exceptional in having an intermediate valence ground state comprised of a mixture of $\mathrm{f}^{0} \mathrm{a}^{\prime 2}$ and $\mathrm{f}^{1} \mathrm{a}^{\prime}$ configurations, where $\mathrm{a}^{\prime}$ is the highest occupied orbital of the $\mathrm{Cp}_{3}$ ligand set. The f electron in the latter occupies the $\cos 3 \phi$ component of the $\operatorname{Ln} 4 f_{ \pm 3}$ orbitals giving a singlet state overall. The uf band intensities are similar to those observed previously in solid state studies but in general are better resolved. The $4 \mathrm{f}^{n-1}$ states are accessed by direct ionization or by a $4 \mathrm{~d}-4 \mathrm{f}$ resonance process coupled with super Koster-Kronig decay. The more intense of the $4 \mathrm{f}^{n}$ states are observable outside the resonance region but become most clearly visible within the resonance region. The mechanisms of their access are discussed. $\mathrm{SmCp}_{3}$ is the most likely candidate to have an intermediate valence ground state as has been established for $\mathrm{YbCp}_{3}$. However, the intensity of the $4 \mathrm{f}^{n}$ bands suggest that an alternative mechanism for their access on $4 \mathrm{f}$ ionization must be present.

\section{References}

1 J. C. Green, Comp. Organomet. Chem., 3, 2006, 1, 381-406.

2 J. C. Green and P. Decleva, Coord. Chem. Rev., 2005, 249, 209.

3 P. A. Cox, Struct. Bonding, 1975, 24, 59.

4 J. N. Chazaviel, M. Campagna, G. K. Wertheim and P. Y. Schmidt, Solid State Commun., 1976, 19, 725.

5 N. Beatham, P. A. Cox, A. F. Orchard and I. P. Grant, Chem. Phys. Lett., 1979, 63, 69.

6 M. Coreno, S. M. de, R. Coates, M. S. Denning, R. G. Denning, J. C. Green, C. Hunston, N. Kaltsoyannis and A. Sella, Organometallics, 2010, 29, 4752-4755.

7 J. J. Yeh and I. Lindau, At. Data Nucl. Data Tables, 1985, 32, 1.

8 U. Fano, Phys. Rev., 1961, 124, 1866.

9 U. Fano and J. W. Cooper, Phys. Rev. A, 1965, 137, 1364.

10 J. W. Allen, L. I. Johansson, I. Lindau and S. B. Hagstrom, Phys. Rev. B: Condens. Matter, 1980, 21, 1335-1342.

11 S. Hüfner, J. Phys. F: Met. Phys., 1986, 16, L31.

12 S. Hüfner, F. Schumann, E. Rotenberg, J. Tobin, S.-H. Yang, B. S. Mun, S. Morton, J. Schäfer and D. Ehm, Phys. Rev. B: Condens. Matter, 2001, 63, 85106.

13 K. C. Prince, R. R. Blyth, R. Delaunay, M. Zitnik, J. Krempasky, J. Slezak, R. Camilloni, L. Avaldi, M. Coreno, G. Stefani, C. Furlani, M. de Simone and S. Stranges, J. Synchrotron Radiat., 1998, 5, 565.

14 M. Coreno, M. de Simone, J. C. Green, N. Kaltsoyannis, N. Narband and A. Sella, Chem. Phys. Lett., 2006, 432, 17-21.

15 R. Coates, M. Coreno, S. M. De, J. C. Green, N. Kaltsoyannis, A. Kerridge, N. Narband and A. Sella, Dalton Trans., 2009, 5943-5953.

16 R. G. Denning, J. Harmer, J. C. Green and M. Irwin, J. Am. Chem. Soc., 2011, 133, 20644-20660.

17 J. M. Birmingham and G. Wilkinson, J. Am. Chem. Soc., 1956, 78, 42 .

18 R. R. Blyth, R. Delaunay, M. Zitnik, R. Krempasky, J. Krempaska, J. Slezak, K. C. Prince, R. Camilloni, L. Avaldi, M. Coreno, G. Stefani, C. Furlani, M. de Simone, S. Stranges and M.-Y. Adam, J. Electron Spectrosc. Relat. Phenom., 1999, 101-103, 959.

19 S. ADF2012.01, Theoretical Chemistry, Vrije Universiteit, Amsterdam, The Netherlands, http://www.scm.com, 2012.

20 W. C. Martin, R. Zalubas and L. Hagan, Atomic Energy Levels \{The Rare Earth Elements, NSRDS-NBS 60, U.S. Government Printing Office, Washington, DC, 1978.

21 H.-D. Amberger, H. Reddmann, T. J. Mueller and W. J. Evans, Organometallics, 2010, 29, 1368-1373.

22 D. A. Wensky and W. G. Moulton, J. Chem. Phys., 1970, 53, 423-435. 
23 H.-D. Amberger, H. Reddmann, T. J. Mueller and W. J. Evans, J. Organomet. Chem., 2011, 696, 2829-2836.

24 M. Karbowiak and C. Rudowicz, Chem. Phys., 2011, 383, 68-82.

25 J. N. Chazalviel, M. Campagna, G. K. Wertheim and P. H. Schmidt, Phys. Rev. B: Condens. Matter, 1976, 14, 4586-4592.

26 M. Campagna, E. Bucher, G. K. Wertheim and L. D. Longinotti, Phys. Rev. Lett., 1974, 33, 165-168.
27 P. Wang, J.-F. Ni, H.-N. Li, W.-H. Zhang and J.-F. Zhu, Surf. Sci., 2008, 602, 3728-3732.

28 J. B. Gruber, B. Zandi and M. F. Reid, Phys. Rev. B: Condens., 1999, 60, 15643-15653.

29 N. Kaltsoyannis, Inorg. Chem., 2013, 52, 3407.

30 C.-H. Wong, T.-Y. Lee and Y.-T. Lee, Acta Crystallogr., Sect. B: Struct. Crystallogr. Cryst. Chem., 1969, 25, 2580.

31 S. H. Eggers, J. Kopf and R. D. Fischer, Acta Crystallogr., Sect. C: Cryst. Struct. Commun., 1987, 43, 2288-2290. 